DOI 10. 18307/2021. 0523

(C) 2021 by Journal of Lake Sciences

\title{
寒区浅水湖冰生消特征及其影响因素"
}

\author{
解 $飞^{1}$, 张议文 $^{2}$, 卢 鹏 $^{1}$, 曹晓卫 ${ }^{1}$, 祖永恒 ${ }^{1}$, 李志军 ${ }^{1 * *}$ \\ (1: 大连理工大学, 海岸和近海工程国家重点实验室, 大连 116024) \\ (2: 大连理工大学,海洋科学与技术学院,盘锦 124221)
}

\begin{abstract}
摘 要: 基于 2019-2020 期间在盘锦市含章湖利用浮式观测平台开展湖冰原型观测试验,分析不同因素对湖冰变化造 成的影响. 结果表明: $99 \mathrm{~d}$ 冰期内湖冰的生消过程可概述为: 湖泊封冻 $(3 \mathrm{~d})$ 一稳定生长 $(62 \mathrm{~d})$ 一冰厚稳定 $(7 \mathrm{~d})$ 一加速消 融 $(24 \mathrm{~d})$ - 破碎分解 $(3 \mathrm{~d})$. 生长期冰厚的平均增长速率为 $0.4 \mathrm{~cm} / \mathrm{d}$, 最大冰厚为 $30.7 \mathrm{~cm}$; 不同深度 $(5 \sim 17 \mathrm{~cm})$ 冰温对气 温变化的响应存在滞后性, 滞后时间为 $70 \sim 158 \mathrm{~min}$, 冰温与气温的最大相关系数为 $0.52 \sim 0.89$; 降雨过程造成冰面反照率 由 0.22 降至 0.09 , 影响了冰内温度以及冰下 $40 \mathrm{~cm}$ 以内的浅层水温, 但 $14 \mathrm{~mm}$ 的降雨量并未引起表面冰厚增加; 降雪过 程造成冰面反照率由 0.25 升至 0.90 , 同时阻碍了 $5 \mathrm{~cm}$ 以内的浅层冰温对气温变化的响应, 但风速长时间大于 $8 \mathrm{~m} / \mathrm{s}$ 时会 导致冰面积雪被吹散, 冰面重新裸露; 消融期冰厚的衰减过程呈抛物线趋势, 存在显著的加速过程, 融化速率由 $0.3 \mathrm{~cm} / \mathrm{d}$ 逐渐增加到 $2.7 \mathrm{~cm} / \mathrm{d}$; 湖冰生长期的冰底热通量均值为 $4.8 \mathrm{~W} / \mathrm{m}^{2}$; 到消融期增加至 $8.1 \mathrm{~W} / \mathrm{m}^{2}$, 为生长期的 1.7 倍; 太阳辐 射与湖冰边界侧向融化是导致湖冰加速融化的关键因素. 本研究填补了国内湖冰冻融全过程实测资料的空缺, 为湖冰热 力学模型的改进提供了科学支撑.
\end{abstract}

关键词: 湖冰;生消特征;冰底热通量;冰厚;降雨;降雪;浮式观测平台

\section{Characteristics and influencing factors of lake ice growth and decay in a shallow lake from a cold region *}

\author{
Xie Fei ${ }^{1}$, Zhang Yiwen ${ }^{2}$, Lu Peng ${ }^{1}$, Cao Xiaowei ${ }^{1}$, Zu Yongheng ${ }^{1}$ \& Li Zhijun ${ }^{1 * *}$ \\ (1: State Key Laboratory of Coastal and Offshore Engineering, Dalian University of Technology, Dalian 116024, P.R. China) \\ (2: Department of Ocean Science and Technology, Dalian University of Technology, Panjin 124221, P.R.China)
}

\begin{abstract}
Based on the prototype observation experiment of lake ice used a floating observation platform in Lake Hanzhang, Panjin City during 2019-2020, the impact of different factors on the evolution of lake ice was analyzed. The results showed that: the growth and decay processes of lake ice in 99 days can be summarized as follows: freeze up ( 3 d) -stable growth ( 62 d) -ice thickness stability ( $7 \mathrm{~d}$ ) -accelerated decay ( $24 \mathrm{~d}$ ) -break up ( $3 \mathrm{~d}$ ). The average growth rate of ice cover during the growth period was $0.4 \mathrm{~cm} / \mathrm{d}$, and the maximum ice thickness was $30.7 \mathrm{~cm}$. The ice temperature at different depths $(5-17 \mathrm{~cm}) \mathrm{had}$ a lag in response to air temperature changes, the lag time was 70-158 $\mathrm{min}$, and the maximum correlation coefficient between ice temperature and the air temperature was $0.52-0.89$. The rainfall reduced the albedo from 0.22 to 0.09 , which affected the ice temperature and the shallow water temperature within $40 \mathrm{~cm}$ below the ice, but the rainfall of $14 \mathrm{~mm}$ did not increase the ice thickness on the upper surface. The snowfall increased the albedo from 0.25 to 0.90 , and at the same time prevented the shallow ice temperature within $5 \mathrm{~cm}$ from responding to temperature changes, but the wind speed greatly controlled the duration of this effect. However, when the wind speed was greater than $8 \mathrm{~m} / \mathrm{s}$ for a long time, the snow on the surface of the ice cover will be blown away, causing the ice surface to be exposed again. The attenuation processes of ice thickness during the melting period showed a parabolic trend, and there were significant acceleration processes. The melting rate gradually increased from $0.3 \mathrm{~cm} / \mathrm{d}$ to $2.7 \mathrm{~cm} / \mathrm{d}$. The average water-to-ice heat flux of the lake ice during the growth period was $4.8 \mathrm{~W} / \mathrm{m}^{2}$. It increased to $8.1 \mathrm{~W} / \mathrm{m}^{2}$ during the decay period, which was 1.7 times that
\end{abstract}

* 2020-11-09 收稿;2021-02-24 收修改稿.

国家自然科学基金项目(41876213,41922045,42007150,51979024)、工信部高技术船舶科研项目(350631009)和国 家重点研发计划项目 (2018YFA0605901) 联合资助.

** 通信作者;E-mail : lizhijun@ dlut.edu.cn. 
of the growth period. Solar radiation and the lateral melting behaviour of the lake ice boundary were the key factors leading to the accelerated melting of lake ice. This study fills the gap of the measured data of the whole processes of lake freezing and melting in China, and provides scientific support for the improvement of the thermodynamic model of lake ice.

Keywords: Lake ice; growth and decay; water-to-ice heat flux; ice thickness; rainfall; snowfall; floating observation platform

全球湖泊主要集中分布于北半球地区 ${ }^{[1]}$, 受气候影响一半以上的湖泊在冬季将发生冻结 ${ }^{[2]}$. 湖冰的生 消过程受到气象因素 (气温、降雨、降雪、风与太阳辐射) 和水文条件 (水深、面积) 共同作用 ${ }^{[3]}$, 具有复杂的 能量平衡关系与显著的地域性特点 ${ }^{[4]}$. 湖泊发生封冻会引发多种效应, 例如封冻期湖泊的微生物群落丰富 度增加 ${ }^{[5]}$; 冰下水体中叶绿素浓度相较夏天更低 ${ }^{[6]}$; 冰层温升膨胀时产生的冰压力会损害护坡 ${ }^{[7]}$. 考虑到湖 冰的上述重要作用, 目前国内外已利用卫星遥感 ${ }^{[8]}$ 、数值模拟 ${ }^{[9-10]}$ 与物理模型试验 ${ }^{[1]}$ 对湖冰的气候、生态和 工程等问题开展大量研究,但多需利用原型试验的观测数据进行校验 ${ }^{[12-14]}$.

国外已于中高纬度地区开展大量湖冰现场试验, 如 Ariano 和 Brown ${ }^{[15]}$ 发现加拿大安大略省中部 MacDonald 湖、Clear 湖以及 Johnson 湖的湖冰会经历较多冬雨, 主要通过冰上融水重新冻结的方式增加冰厚. Rouse 等 ${ }^{[16]}$ 对比了 Slave 湖和 Bear 湖的湖冰热力特征与地表气候, 结果显示湖泊吸收的太阳辐射是影响冰 期变化的关键因素. Cheng 等 ${ }^{[17]}$ 根据芬兰北部 Orajärvi 湖连续 3 个冬季的湖冰观测数据发现降雨量与降雪 量的模拟对于估算冰雪质量平衡至关重要. Svacina 等 ${ }^{[18]}$ 利用加拿大曼尼托巴省 Malcolm Ramsay 湖冰上冰 雪反照率的原位测量数据, 对湖冰模型反照率参数化进行了评估. 中国寒区湖冰多位于中低纬度地区, 与高 纬度湖泊相比具有其自身特点. 李志军等 ${ }^{[19]}$ 对黑龙江红旗泡水库进行冬季现场观测, 获取了冰面总辐射、 反射、气温、水温与冰厚等数据, 发现每日近表面冰温与气温比值峰值的发生时间比辐射峰值的发生时间滞 后 $1.4 \mathrm{~h}$. 脱友才等 ${ }^{[20]}$ 对丰满水库进行了冰厚原型观测试验, 发现水库封冻期冰厚平均增长率为 $0.9 \mathrm{~cm} / \mathrm{d}$. 黄文峰等 ${ }^{[21]}$ 对高原典型热融湖进行了水温原位观测, 发现水温垂直结构具有显著的年循环过程. Cao 等 ${ }^{[22]}$ 针对乌梁素海探究了太阳辐射在湖冰内部的传输规律, 结果显示湖冰的反照率和透射率主要受积雪和融水 等冰面条件的影响. 汤明光等 ${ }^{[23]}$ 同样于乌梁素海开展湖冰反照率研究, 提出了描述湖冰反照率日变化曲线 的数学模型.

上述湖冰研究为气候研究、冰下水生态研究与水利工程灾害防治奠定了基础 ${ }^{[24]}$, 但受限于湖冰的地域 性特点, 研究结果通常仅能代表小区域内的湖冰特性,多数地区的湖冰变化特征仍为空白. 目前湖冰生消过 程的原型观测考虑到冰面工作的安全性, 需要冰层满足一定承载力后开展, 因此多数观测缺失了湖冰封冻 前期与消融后期的关键数据, 而完整的冰层生消过程对于湖冰变化的理解至关重要. 降雨与降雪等气象条 件对湖冰特征的影响在国内寒区鲜有报道, 仍需加以探究. 本文选取含章湖开展原型观测试验, 该湖泊属于 典型的亚洲东部季风区中纬地区浅水湖泊. 本研究于 2019-2020 年期间利用浮式观测平台与岸基气象塔 获得了湖冰生消的完整过程与水文气象要素, 依此探究寒区浅水湖冰的变化特征, 并重点分析气温、降雨、 降雪、风速、太阳辐射与湖泊空间差异对冰水温度场、冰厚和冰面特征的影响规律, 从而为湖冰热力学模型 的开发与改进提供科学支撑.

\section{1 观测区概况}

含章湖 $\left(40^{\circ} 40^{\prime} 12^{\prime \prime} \sim 40^{\circ} 43^{\prime} 48^{\prime \prime} \mathrm{N}, 122^{\circ} 2^{\prime} 24^{\prime \prime} \sim 122^{\circ} 9^{\prime} 0^{\prime \prime} \mathrm{E}\right.$ ) 位于辽宁省盘锦市辽东湾新区, 近邻营口, 地处 北温带, 属暖温带大陆性半湿润季风气候, $1951-2013$ 年冬季平均气温为 $-6.6^{\circ} \mathrm{C}^{[25]}$. 湖泊总面积 $10 \mathrm{~km}^{2}$, 轴 线为东西方向, 湖岸为立式混凝土墙. 湖泊通过闸门连通渤海, 但外海并不会对湖泊水质产生影响, 属于中 纬度咸水人工湖, 平均水深 $6.0 \mathrm{~m}$. 根据现场测量, 冬季冰下湖水平均含盐量为 $6.9 \mathrm{~g} / \mathrm{L}$, 且并无显著垂向盐度 差异, 水体冰点约为 $-0.37^{\circ} \mathrm{C} .2019-2020$ 年冬季在该湖开展现场试验的研究区及相关仪器布置情况如图 1 所示, 浮式观测平台 (红色五角星标记) 与岸基气象塔 (红色三角星标记) 均布置于含章湖东南侧, 湖冰观测 点处水深为 $3.7 \mathrm{~m}$. 桥与河口 (红色虚线处) 分别在浮式观测平台东南侧与岸基气象塔北侧, 河口北侧 $1.5 \mathrm{~km}$ 处存在人水口. 


\section{2 研究方法}

浮式观测平台搭载铂电阻冰水温度链 ${ }^{[22]}$ (距离水面垂直深度依次为 $0.05 、 0.08 、 0.11 、 0.14 、 0.17 、 0.23$ 、 $0.29 、 0.40 、 0.55 、 1.05 、 2.05 、 3.05 、 3.70 \mathrm{~m}$, 精度均为 $0.1^{\circ} \mathrm{C}$ ) 、水下超声波测距仪 (冰厚测量量程 $0.1 \sim 0.5 \mathrm{~m}$, 精 度为 $0.01 \mathrm{~m}$ ) 、环温传感器 (精度为 $0.1^{\circ} \mathrm{C}$ )、环湿传感器、太阳总辐射表、太阳反辐射表和冰面环境监控仪. 数 据采集间隔均设定为 $1 \mathrm{~min}$,通过 GPRS 无线传输方式实时获取数据. 该浮台于 2019 年 12 月 7 日布置于湖 中, 观测点初始冰厚为 $5 \mathrm{~cm}$; 于 2020 年 3 月 6 日浮台结束工作, 撤站时湖冰已完全消融. 其中自 2 月 1 日开 始, 由于疫情影响了现场人员对太阳能电池板的维护, 观测数据在夜间和阴天存在丢失现象, 缺失的日均气 象数据 (气温、湿度与风速) 由国家气象数据中心提供的中国地面气候资料日值数据集补充 ( http://data. cma.cn). 通过校准, 最终选择营口站提供的数据集进行补充. 岸基气象塔负责风速、太阳总辐射与岸冰反辐 射的观测. 风速探头与冰面垂直距离为 $5 \mathrm{~m}$, 数据时间范围为 2019 年 12 月 16 日-2020 年 1 月 20 日, 采集 间隔设定为 $1 \mathrm{~min}$.
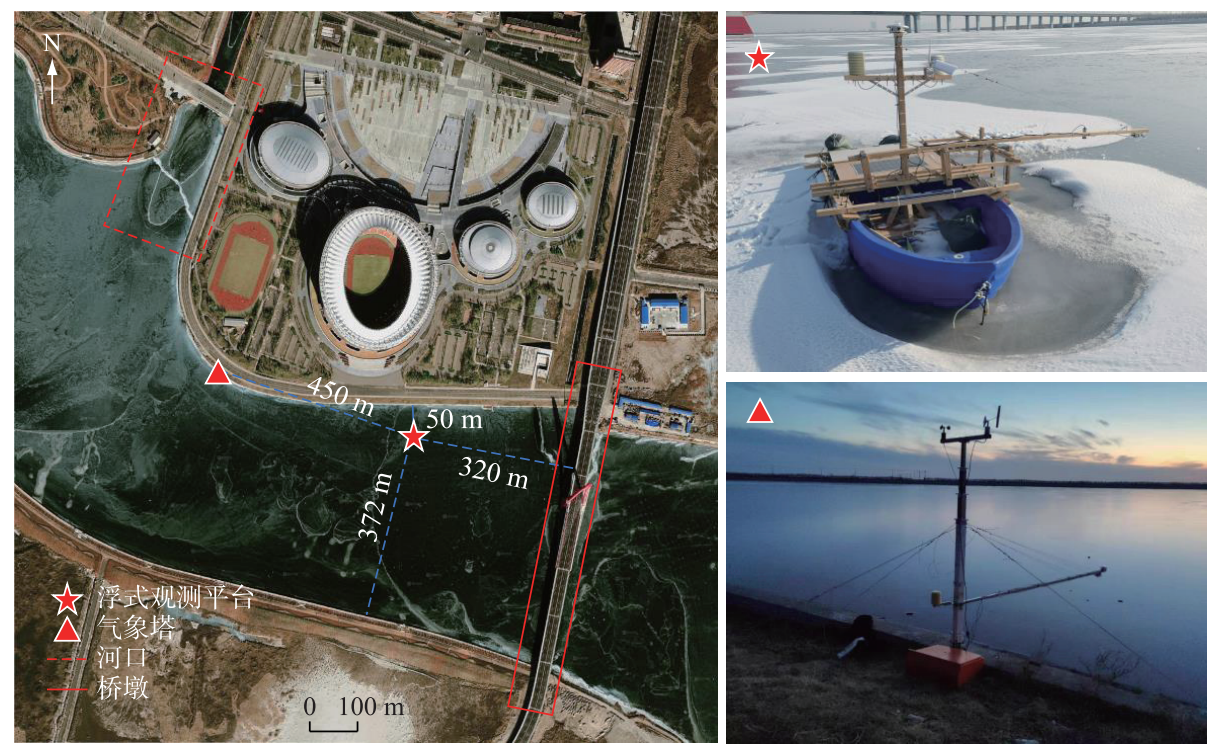

图 1 研究区、浮式观测平台与气象塔

Fig.1 Research area, floating observation platform and weather station

\section{3 结果}

\section{1 湖冰生消过程}

现场观测期间, 通过布置在岸边和观测平台上的摄像系统, 对湖冰的生消过程进行了连续的可视化观 测 ,不同日期拍摄的湖冰生消过程如图 2 所示.

从图 2 可以看到, 12 月 5 日有近 70\% 的湖面封冻, 岸边冰厚 $1 \mathrm{~cm}$, 剩余开阔水域主要存在于浮式观测平 台东南侧桥墩下与气象塔北侧河口处; 12 月 6 日 90\%湖面封冻, 仅东南侧桥墩下存在开阔水域; 12 月 7 日浮 式观测平台放置于湖中, 北侧岸边冰厚 $3 \mathrm{~cm}$, 浮台处冰厚 $5 \mathrm{~cm} ; 12$ 月 8 日浮台完全冻结于湖中开展观测工 作. 12 月 22 日发生降雪导致冰面被积雪覆盖, 而东南侧桥墩下依然存在开阔水域; 冰上覆雪状态持续到 12 月 29 日,该日持续的强风吹散大量积雪, 12 月 30 日大面积湖冰呈裸露状态. 1 月 5 日岸冰冰面形态与湖冰 存在显著差异, 湖冰冰层相对岸冰更加透明. 1 月 6 日的降雪量更大,但随后的强风使得 1 月 8 日冰面重新 裸露. 2 月 25 日东南侧桥墩下开阔水域已显著向西北侧延伸. 3 月 6 日浮台处已完全为开阔水域,但气象塔 西南侧湖面仍为封冻状态; 3 月 8 日气象塔西南侧开始出现开阔水域, 且方向自西向东延伸; 3 月 11 日浮台 


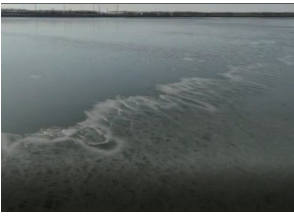

2019-12-05

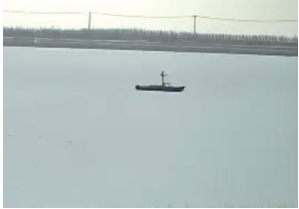

2019-12-29

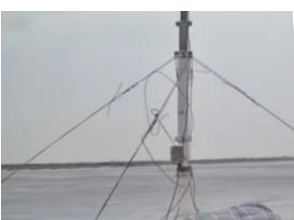

2020-03-06

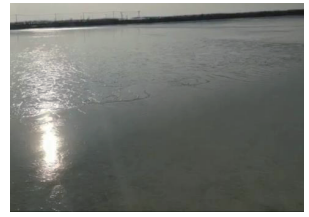

2019-12-06

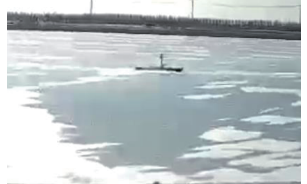

2019-12-30

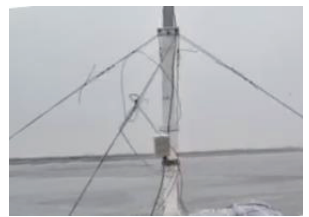

2020-03-08

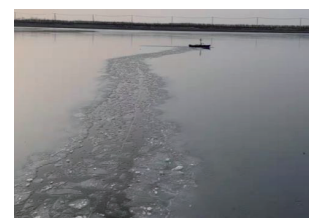

2019-12-08

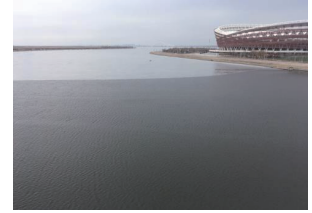

2020-02-25

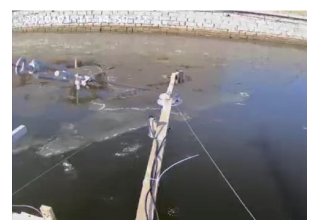

2020-03-11

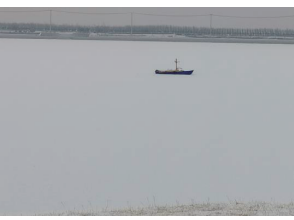

2019-12-22

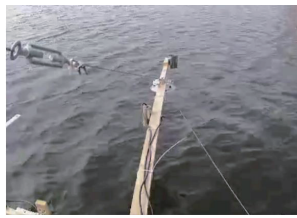

2020-03-06

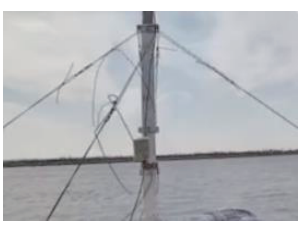

2020-03-12

图 2 岸基和浮台上布置的摄像头监测到的湖冰生消过程

Fig.2 The growth and decay processes of lake ice monitored by cameras arranged on the shore base and floating platform

周围再次出现破碎的浮冰,至 3 月 12 日气象塔西南侧完全变为开阔水域. 综上,含章湖 2019-2020 年冰期 共 $99 \mathrm{~d}$,其中湖泊由开阔水到 $90 \%$ 封冻历时 $3 \mathrm{~d}$,之后湖冰生长过程 $69 \mathrm{~d}$, 融化过程 $27 \mathrm{~d}$.

\section{2 气象要素、冰水温度场及冰层生消过程}

现场观测得到的气象要素、冰水温度场及冰层生消过程如图 3 所示.

$12 、 1$ 与 2 月的平均气温分别为 $-3.93 、-5.63$ 与 $-2.58^{\circ} \mathrm{C}$ ( 图 3a), 均高于 $1981-2010$ 年间对应的月平均 值 $\left(-5.26 、-8.27\right.$ 与 $\left.-4.20^{\circ} \mathrm{C}\right)$. 其中, 12 月 4 日 -12 月 6 日的日均气温较低, 分别为 $-6.6 、-12.3$ 与 $-5.3^{\circ} \mathrm{C}$, 此 时期是湖泊发生封冻转换的主要阶段; 12 月 8 日- 12 月 16 日的日均气温在 $0^{\circ} \mathrm{C}$ 上下波动, 随后降为 $0^{\circ} \mathrm{C}$ 以 下; 2 月 18 日-3 月 6 日气温正负交替,最高气温仅为 $5.1^{\circ} \mathrm{C}$. 环境湿度的变化过程与气温近似,但日波动更 剧烈 (图 3b), 较高的气温会引起较高的湿度. 风速日波动较大, 在 12 月 17 日、12 月 21 日、12 月 31 日与 1 月 8 日均记录到高于 $10 \mathrm{~m} / \mathrm{s}$ 的瞬时风速 (图 3c). 整个冬季人射太阳辐射的每日峰值存在先减小后增加的 趋势, 最小值出现在 12 月中旬 (约 $600 \mathrm{~W} / \mathrm{m}^{2}$ ), 随后逐渐升高到 $800 \mathrm{~W} / \mathrm{m}^{2}$. 冰面反辐射强度在冰面覆雪期 间较高 (约 $300 \mathrm{~W} / \mathrm{m}^{2}$ ), 在裸冰及冰面融化期间较低,仅为 $50 \mathrm{~W} / \mathrm{m}^{2}$ 左右 (图 $3 \mathrm{~d}$ ).

从图 3e 中可以看到, 冰厚于 12 月 5-7 日由 $0 \mathrm{~cm}$ 迅速发展至 $5 \mathrm{~cm}$, 后因气温回升至 $0^{\circ} \mathrm{C}$ 而暂停生长. 这一过程中, $-12.3^{\circ} \mathrm{C}$ 的气温与 $2^{\circ} \mathrm{C}$ 的水温形成的巨大温度梯度是薄冰出现并迅速发展的重要原因, $1.7 \mathrm{~cm} / \mathrm{d}$ 的生长速率也是整个观测期记录到的冰厚最快发展过程. 12 月 17 日一 2 月 6 日冰厚稳定增长,生长速率稳 定在 $0.45 \mathrm{~cm} / \mathrm{d} .2$ 月 7 日 -2 月 13 日冰厚变化缓慢 $( \pm 0.13 \mathrm{~cm})$, 此时冰下 $25 \mathrm{~cm}$ 处出现 $1^{\circ} \mathrm{C}$ 的暖水层, 其传 热作用抵消了负积温对冰水界面的冻结作用. 2 月 14 日- 3 月 4 日观测点处的冰厚迅速消融, 同时伴随暖水 层位置向上延伸, 消融速率由 $0.3 \mathrm{~cm} / \mathrm{d}$ 逐渐增至 $2.7 \mathrm{~cm} / \mathrm{d}$. 整个观测期, 冰厚平均增长速率约为 $0.44 \mathrm{~cm} / \mathrm{d}$, 平均消融速率为 $1.3 \mathrm{~cm} / \mathrm{d}$, 最大冰厚为 $30.7 \mathrm{~cm}$.

需要注意的是图 3e 中红色圆圈处,虽然冰正处于生长期,但 12 月 30 日的冰厚日均值减小了约 $0.5 \mathrm{~cm}$. 结合实测数据发现, 12 月 29 日的日均气温为 $1.4^{\circ} \mathrm{C}$, 深度 $20 \sim 40 \mathrm{~cm}$ 的水层短时期内出现了 $2^{\circ} \mathrm{C}$ 的暖水层, 同时伴随着强风产生的风吹雪作用,冰面失去了雪层绝热作用的保护. 此时冰层上下界面的温度都高于冰 点, 即使是在生长期冰层底部仍会发生融化. 随着 12 月 30 日的日均气温突降至 $-12.7^{\circ} \mathrm{C}$, 冰层底部又发生了 
约 $1 \mathrm{~cm}$ 的快速增长. 冰下水温在冻结期和融化期表现出了不同的特征 (图 3e). 12 月 7 日-2 月 5 日冰下水 温介于 $0 \sim 2^{\circ} \mathrm{C}$ 之间, 保持在均匀混合状态. 自 2 月 6 日开始,随着湖冰融化, $55 \mathrm{~cm}$ 深度附近的水层温度逐渐 升高,且暖水层距冰底的距离从 $25 \mathrm{~cm}$ 逐渐减少到 $5 \mathrm{~cm}, 3$ 月 6 日湖水温度已介于 $2 \sim 6^{\circ} \mathrm{C}$ 之间.
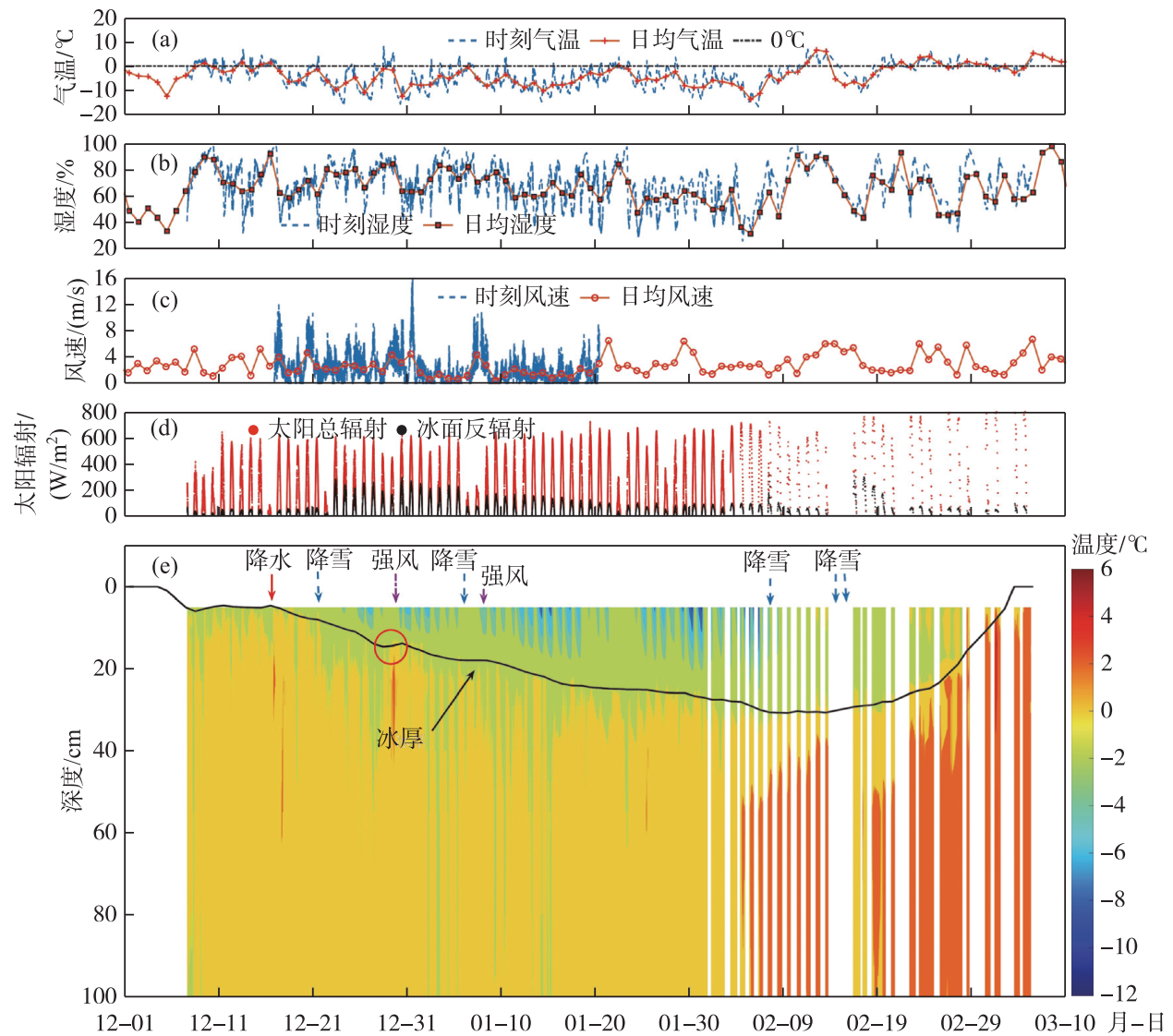

图 3 气象要素、冰水温度场和冰层生消过程 ( $\mathrm{a}:$ 气温; $\mathrm{b}:$ 湿度; $\mathrm{c}:$ 风速 $; \mathrm{d}:$ 太阳辐射; $\mathrm{e}$ :冰水温度场与冰厚)

Fig.3 Meteorological elements, temperature field of ice (water) and the growth and decay processes of ice cover ( a: air temperature; b: humidity; $\mathrm{c}$ : wind velocity; $\mathrm{d}$ : solar radiation; e: temperature field and ice thickness)

\section{4 讨论}

\section{1 气温对湖冰变化的影响}

气温是影响湖冰发展的关键因素 ${ }^{[26]}$. 对观测期内冰厚变化过程与气一冰温度传递规律进行分析, 结果 如图 4 所示.

冰厚的生长过程呈线性趋势, 日均冰厚生长速率约为 $0.4 \mathrm{~cm} / \mathrm{d}$, 结合气温变化过程 (图 3a) 可知, 在冰厚 生长期气温是主要驱动因子. 引入冻冰度日的概念, 利用 Stafen 方程来描述冰厚的增长过程, $h=2.23$ $(F D D)^{0.5}-12.4, R^{2}=0.993, P<0.0001$. 回归系数 $\alpha=2.23$ 接近于李志军等 ${ }^{[19]}$ 的研究结果 (在少风有雪条件 下, $\alpha=2.24)$. 冰厚的消融过程近似于抛物线, 消融速率由 $0.3 \mathrm{~cm} / \mathrm{d}$ 逐渐增加到 $2.7 \mathrm{~cm} / \mathrm{d}$, 存在显著的加速 过程. 在此期间, 气温并未对冰厚变化产生显著贡献 (图 3a), 冰厚的快速衰减行为主要受太阳辐射及其他 因素控制, 将在 4.4 节讨论.

为研究气温对冰内温度场的影响,选取 1 月 8 日- 1 月 30 日冰面无积雪时的气温与冰温数据计算不同 

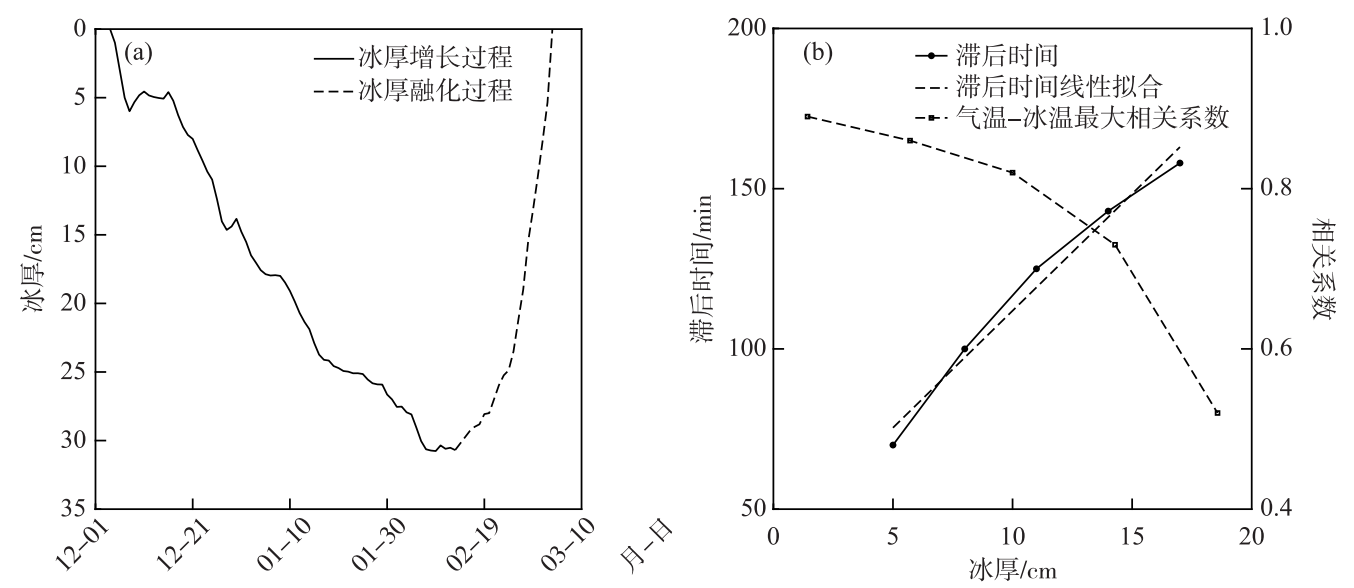

图 4 冰厚变化过程 $(\mathrm{a})$ 和冰温响应气温变化的滞后效应 $(\mathrm{b})$

Fig.4 Evolution of ice thickness (a) and the hysteresis effect of ice temperature in response to air temperature change $(b)$

深度冰温时间序列相对气温时间序列的滞后相关系数, 根据最大相关系数的时间相位差作为滞后时间 ${ }^{[27]}$ :

$$
\begin{gathered}
R_{\mathrm{c}}\left[T_{\mathrm{a}}\left(t_{1}\right), T_{i}\left(t_{2}\right)\right]=\frac{\sum\left[T_{\mathrm{a}}\left(t_{1}\right)-\overline{\left.T_{\mathrm{a}}\left(t_{1}\right)\right]}\left[T_{i}\left(t_{2}\right)-\overline{\left.T_{i}\left(t_{2}\right)\right]}\right.\right.}{\sqrt{\sum\left[T_{\mathrm{a}}\left(t_{1}\right)-\overline{T_{\mathrm{a}}\left(t_{1}\right)}\right]^{2} \sum\left[T_{i}\left(t_{2}\right)-\overline{T_{i}\left(t_{2}\right)}\right]^{2}}} \\
R_{\mathrm{cmax}}\left[T_{\mathrm{a}}\left(t_{1}\right), T_{i}\left(t_{2}\right)\right]=\operatorname{MAX}\left\{R_{\mathrm{c}}\left[T_{\mathrm{a}}\left(t_{1}\right), T_{i}\left(t_{2}\right)\right]\right\} \\
\Delta t_{i}=t_{2}-t_{1} \quad \Delta t_{i}=\{1,2,3, \cdots(\min )\}
\end{gathered}
$$

式中, $T_{\mathrm{a}}\left(t_{1}\right)$ 与 $T_{i}\left(t_{2}\right)$ 分别为时间序列内的气温平均值与冰温平均值 (冰温对应的冰内深度为 $i, i=5 、 8 、 11$ 、 $14 、 17 \mathrm{~cm})$, 得到相关系数 $R_{\mathrm{c}}\left[T_{\mathrm{a}}\left(t_{1}\right), T_{i}\left(t_{2}\right)\right]$. 选取 $R_{\mathrm{c}}$ 中最大值 $\operatorname{MAX}\left\{R_{\mathrm{c}}\left[T_{\mathrm{a}}\left(t_{1}\right), T_{i}\left(t_{2}\right)\right]\right\}$ 所对应的 $\Delta t_{i}$ 为 滞后时间, 计算过程中 $t_{1}$ 不变, 逐渐延后 $t_{2}$. 如图 $4 \mathrm{~b}$ 显示, $5 \mathrm{~cm}$ 深度冰温响应气温变化的滞后时间为 $70 \mathrm{~min}$, 最大相关系数为 0.89 ; 而 $17 \mathrm{~cm}$ 深度冰温响应气温变化的滞后时间为 $158 \mathrm{~min}$, 最大相关系数为 0.52 . 对冰厚 与滞后时间的拟合公式为:

$$
\Delta t_{i}=7.3 Z_{i}+38.9 \quad\left(17 \mathrm{~cm} \geqslant Z_{i} \geqslant 5 \mathrm{~cm}\right)
$$

式中, $\Delta t_{i}$ 为滞后时间, $\min ; Z_{i}$ 为冰内深度, $\mathrm{cm}$. 结果表明冰温相对气温的相关性随冰层深度的增加逐渐降低, 滞后时间逐渐延长, 且滞后时间与冰层厚度之间存在一定的线性关系 $\left(R^{2}=0.98, P=0.0019\right)$. 该公式在冰温 观测深度范围内 $\left(17 \mathrm{~cm} \geqslant Z_{i} \geqslant 5 \mathrm{~cm}\right)$ 适用. 雷瑞波等 ${ }^{[27]}$ 的研究发现中山站附近海冰对气温高频波动的衰减 效应要明显强于湖冰, 且二者都遵循滞后时间随深度增加接近线性 $(P<0.01)$ 的发展趋势. 本研究中得到浅 层冰温与气温的最大相关系数要大于雷瑞波等 ${ }^{[27]}$ 在南极中山站得到的研究结果, 滞后时间也要小于雷瑞波 等 ${ }^{[27]}$ 的结果. 结合试验环境可知, 后者研究针对于厚度 $66 \mathrm{~cm}$ 与 $102 \mathrm{~cm}$ 的极地湖冰, 远大于本研究中的湖 冰厚度,而冰层的垂向热传导通量又主要与气温和冰厚有关. 相同气温条件下,较薄的冰厚会导致冰内垂向 热传导通量更大, 冰温响应气温变化的滞后时间也更短. 故研究区气温与冰厚的差异是造成本研究中滞后 时间小于雷瑞波等 ${ }^{[27]}$ 结果的主要原因.

\section{2 降雨对湖冰变化的影响}

整个观测期内, 仅在 12 月 16 日发生了 1 次 $14 \mathrm{~mm}$ 的降雨事件,降雨前后的气象因素和水温数据如图 5 所示.

降雨前 (12 月 $14 、 15$ 日)气温在 $0^{\circ} \mathrm{C}$ 上下波动 (图 5a), 相对湿度介于 $50 \% \sim 80 \%$ 之间, 冰厚维持在 $5 \mathrm{~cm}$ 左右; 浮台观测点处湖冰反照率为 0.16 , 岸冰反照率为 0.28 , 岸冰反照率略高 (图 $5 b$ ), 存在约 0.1 的差值. 因 观测点处湖冰几乎都由柱状冰组成, 相对于岸冰更加透明, 而颗粒冰 (岸冰) 反照率要高于柱状冰 ${ }^{[18]}$, 因此 在裸冰状态下表面包含颗粒冰较多的岸冰拥有更高的反照率; 冰下水温较均匀 (图 5c), 深层水温 (40 360 
$\mathrm{cm}$ ) 要略低于浅层水温 $(5 \sim 40 \mathrm{~cm}) .12$ 月 16 日白天发生不连续的降雨事件, 持续到晚上 $6: 00$, 共计雨量 14 $\mathrm{mm}$. 气温几乎维持在 $0^{\circ} \mathrm{C}$ (图 5a), 而相对湿度逐渐升高, 峰值接近于 $100 \%$, 说明降雨的发生显著抬高了冰 上环境中的湿度. 湖冰反照率与岸冰反照率均发生了显著衰减的变化 (图 5b), 降雨前的湖冰与岸冰反照率 分别为 0.16 与 0.28 , 降雨后的湖冰与岸冰反照率分别为 0.08 与 0.10 , 平均反照率受降雨影响由 0.22 降至 0.09. 随着冰面积水, 粒状冰和柱状冰的表观光学性质微小差异被积水影响所掩盖. 冰面有薄层积水时, 冰 面反照率由水层和冰层联合作用决定, 但水层起主导作用 ${ }^{[28]}$; 降雨过程造成了 $5 \sim 20 \mathrm{~cm}$ 的水层温度趋于 $0^{\circ} \mathrm{C}$, 降雨停止后浅层水温 $(5 \sim 40 \mathrm{~cm}$ ) 发生了整体的温度升高. 一方面由于垂直结构气一水一冰一水的热交换 行为, 另一方面由于温暖的雨水经湖一岸处汇入湖水, 带来少量横向的热量; 反观深层水温 $(40 \sim 360 \mathrm{~cm})$, 并 未发生显著的升高过程.

降雨结束后 (12月 17 日)气温逐渐降至负温 (图 5a), 相对湿度降至 50\%左右, 湖冰与岸冰的反照率再 次升高 (图 5b), 近似于 12 月 14 日的状态; 冰下水温受温度梯度影响逐渐混合, 但浅水层相较深水层而言混 合更剧烈. 由于降雨量仅 $14 \mathrm{~mm}$, 冰面积水二次冻结造成的冰厚增加不足 $1.5 \mathrm{~cm}$, 冰厚的发展仍然主要由冰 底的变化决定,这与 Ariano 等 ${ }^{[15]}$ 的研究结果不同. Ariano 等的研究区冬季降雨量更高 $(580 \mathrm{~mm})$, 使得冰面 积水冻结发生了显著的冰增长, 对整个冰厚的发展贡献较大.

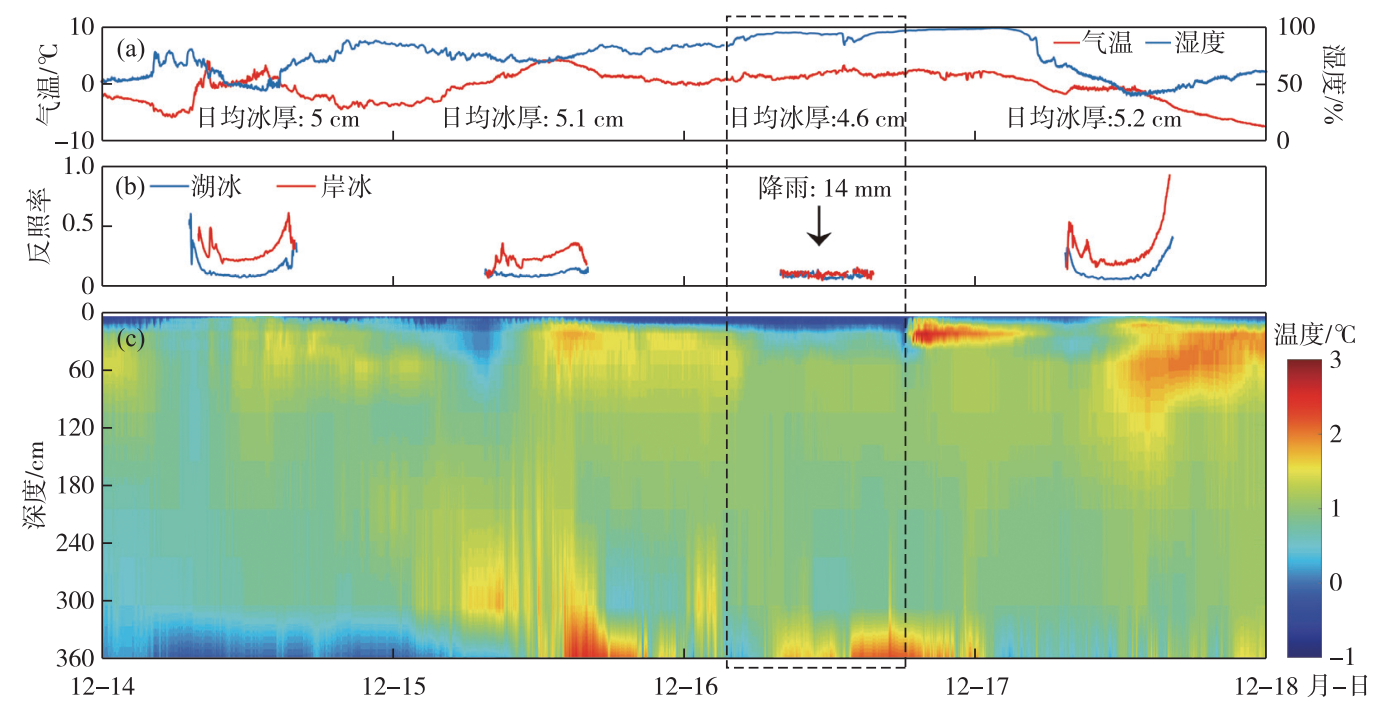

图 5 降雨前后气象因素、反照率和水温 ( $\mathrm{a}$ :气温与湿度; $\mathrm{b}$ : 湖冰和岸冰反照率; $\mathrm{c}$ : 水温)

Fig.5 Meteorological factors, albedo and water temperature before and after rainfall ( $\mathrm{a}$ : air temperature and humidity; b: albedo of lake ice and shore ice; $\mathrm{c}$ : water temperature)

\section{3 降雪对湖冰变化的影响}

观测期内共发生 4 次降雪事件, 分别在 12 月 22 日、 1 月 6 日、 2 月 7 日和 2 月 15 日,后两次降雪事件数 据缺失, 图 6 给出了前两次降雪前后的风速、反照率及气温与 $5 \mathrm{~cm}$ 层冰温.

12 月 22 日发生了整个观测期的第 1 次降雪, 积雪厚度为 $3.5 \mathrm{~cm}(06: 00-12: 00)$. 随着积雪覆盖于冰 面, 整个湖冰与大气之间的热交换模式转换为气一雪冰一水的垂直结构. 12 月 25 日与 12 月 26 日的风速介 于 $2 \sim 8 \mathrm{~m} / \mathrm{s}$ 之间; 与之类似的是 12 月 29 日与 12 月 30 日的风速介于 $3 \sim 8 \mathrm{~m} / \mathrm{s}$ 之间, 且持续时间较长 (图 $6 \mathrm{a}$ ), 这次风吹雪过程直接导致大面积的湖冰裸露出来(图 2). 1 月 6 日发生了整个观测期的第 2 次降雪 (04:00-12:00), 积雪厚度为 $6 \mathrm{~cm}$, 而 1 月 7 日与 1 月 8 日的风速介于 $2 \sim 10 \mathrm{~m} / \mathrm{s}$ 之间, 拥有较高的风速与 较长的持续时间 (图 6a). 虽然第 2 次降雪的雪量更大,但在高风速环境下对冰面造成的覆雪时间却更短 ( 图 2).

结合冰面反照率可知 (图 6b), 12 月 22 日降雪后, 岸冰和湖冰反照率均迅速由 0.25 升高至 0.90 ; 次日岸 
冰反照率下降至 0.70 左右, 湖冰反照率降至 0.50 . 这种差异主要是由于湖岸处的立墙使得更多的新鲜积雪 被堆积到岸边, 导致近岸冰的反照率更高. 随后在冰面积雪变质作用下, 两处的反照率逐日降低. 需要注意 12 月 26 日岸冰反照率再次显著升高, 而湖冰反照率也停止降低. 因为该日的风力 (图 6a) 并未完全吹散冰 面积雪, 但却导致了积雪的重新分配, 造成湖冰处的反照率近乎不变, 而岸冰处受积雪增加影响反照率升 高; 类似的现象还出现在 12 月 29 日与 12 月 30 日, 特别的是 12 月 29 日的湖冰与岸冰反照率瞬间降低, 又 迅速于 12 月 30 日升高. 第 1 次反照率降低是因为显著的风吹雪导致整个湖冰的大面积裸露, 第 2 次反照率 升高则是因为风应力的继续作用致使冰面反辐射记录表的下垫面存在被带来的新鲜积雪. 观测期第 2 次降 雪造成的冰面反照率变化过程更加明显,从 1 月 6 日湖冰反照率与岸冰反照率的显著升高, 再受风应力控制 发展到 1 月 7 日与 1 月 8 日的反照率迅速衰减(图 6a). 冰面反照率的变化直接影响太阳辐射对整个湖冰的 热量传导效应,进而干扰了整个湖冰的发展.
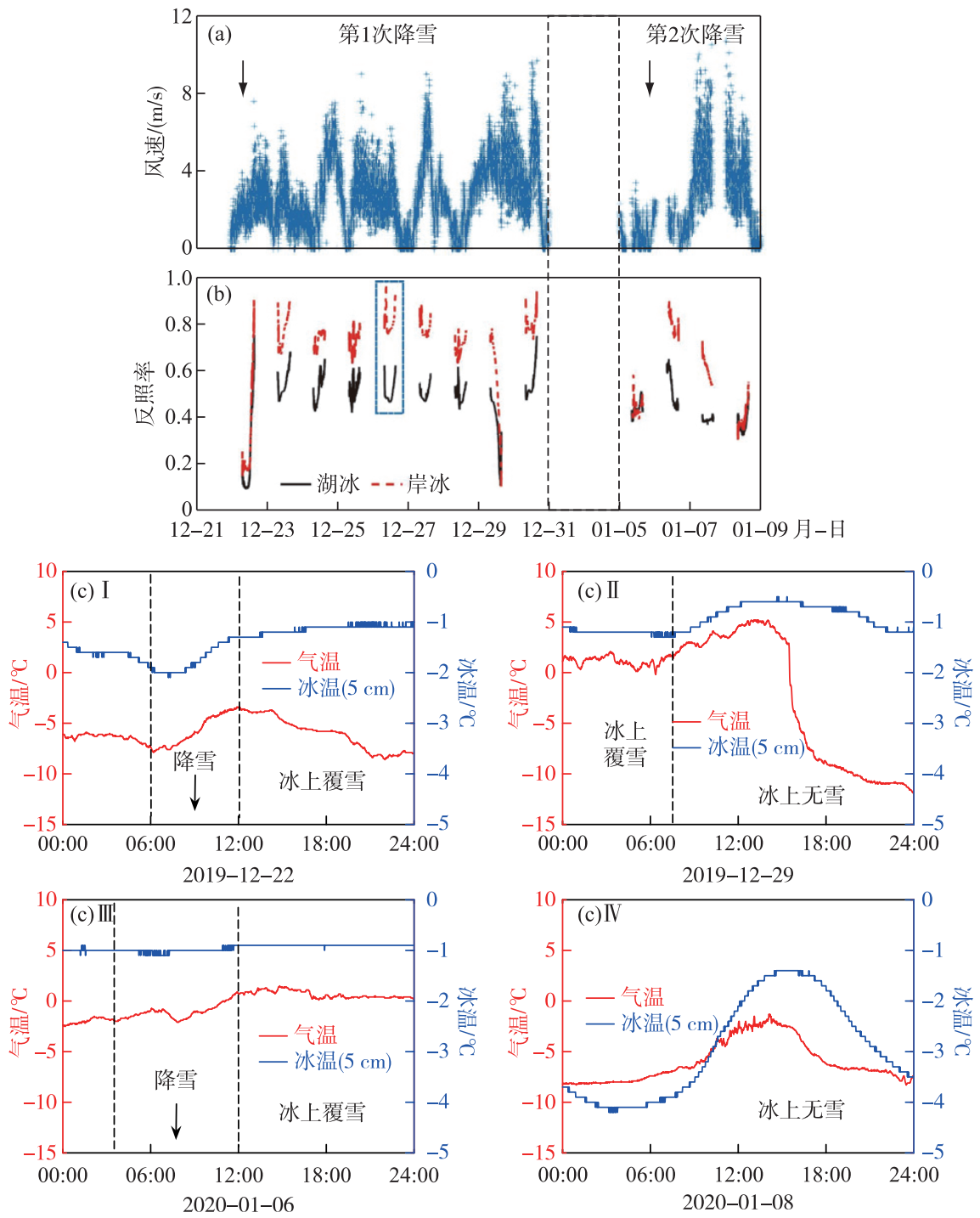

图 6 风速 $(\mathrm{a})$ 、反照率 $(\mathrm{b})$ 、气温与 $5 \mathrm{~cm}$ 层冰温 $(\mathrm{c})$

Fig.6 Wind speed (a), albedo (b), air temperature and $5 \mathrm{~cm}$ ice temperature (c) 
随着降雪事件的发生, 冰上积雪逐渐累积, 由于积雪较低的热传导特性, 湖冰与大气之间不再存在直接 的热交换过程. 由图 6c I 可知,裸冰状态下 $5 \mathrm{~cm}$ 深度的冰温波动与气温变化近似,但随着 12 月 22 日发生 降雪并逐渐累积, 气温降低后冰温不再显著响应于气温变化; 随着冰上积雪被吹散, 冰温重新响应于气温变 化 (图 6c II ); 近似的现象出现于 1 月 6 日 (图 6c III), 但因气温较为稳定导致冰温的弱响应现象不明显; 1 月 8 日 (图 6c IV) 裸冰状态下的 $5 \mathrm{~cm}$ 深度冰温响应气温变化存在显著的滞后性 (图 $4 \mathrm{~b}$ ). 对比图 $3 \mathrm{e}$ 可知,降雪/ 积雪过程对冰下水温结构并未产生显著影响. 不同于降雨事件的快速响应,降雪过程对于湖冰的影响表现 为持续性. 积雪厚度与变质过程都干扰了大气与湖冰之间的能量交换以及太阳辐射在冰内的穿透能量, 同 时风应力控制了冰面积雪的重新分配行为,决定了积雪对湖冰作用的持续时间.

\section{4 影响湖冰融化的关键因素}

相较于湖冰生长期而言, 湖冰消融期的融化过程更为复杂 ${ }^{[29]}$. 图 7 给出了 2 月 1 日-3 月 6 日消融期的 湖冰特征.

自 2 月 20 日冰上积雪消失后湖冰反照率一直保持在较低水平,约 0.2 (图 7a), 且一直持续到浮台观测 点处湖冰完全消融. 较低的反照率使得更多的太阳辐射被湖冰及冰下水体吸收 (图 7b). 考虑到消融期记录 到的太阳辐射数据存在缺失现象, 以湖冰及冰下水体吸收的太阳辐射峰值衡量其对冰厚衰减的贡献能力. 在湖冰生长及稳定期, 2 月 1 日- 13 日之间湖冰及冰下水体吸收的太阳辐射能量峰值 $F_{G-m a x}$ 为 $621 \mathrm{~W} / \mathrm{m}^{2}$; 而 在湖冰消融期, 2 月 14 日- 3 月 6 日之间湖冰及冰下水体吸收的太阳辐射能量峰值 $F_{\mathrm{D}-\mathrm{max}}$ 则为 $796 \mathrm{~W} / \mathrm{m}^{2}$. 结 合图 7c 中的气温数据可知, 2 月 1 日- 13 日之间的日均气温 $T_{\mathrm{G}-\mathrm{A}}$ 为 $-4.2^{\circ} \mathrm{C}$, 而 2 月 14 日 -3 月 6 日之间的日 均气温 $T_{\mathrm{D}-\mathrm{A}}$ 为 $-1.2^{\circ} \mathrm{C}$, 虽有升高但仍低于 $0^{\circ} \mathrm{C}$. 结合图 $7 \mathrm{~d}$ 的冰厚消融过程可知, 湖冰吸收太阳辐射能量增加

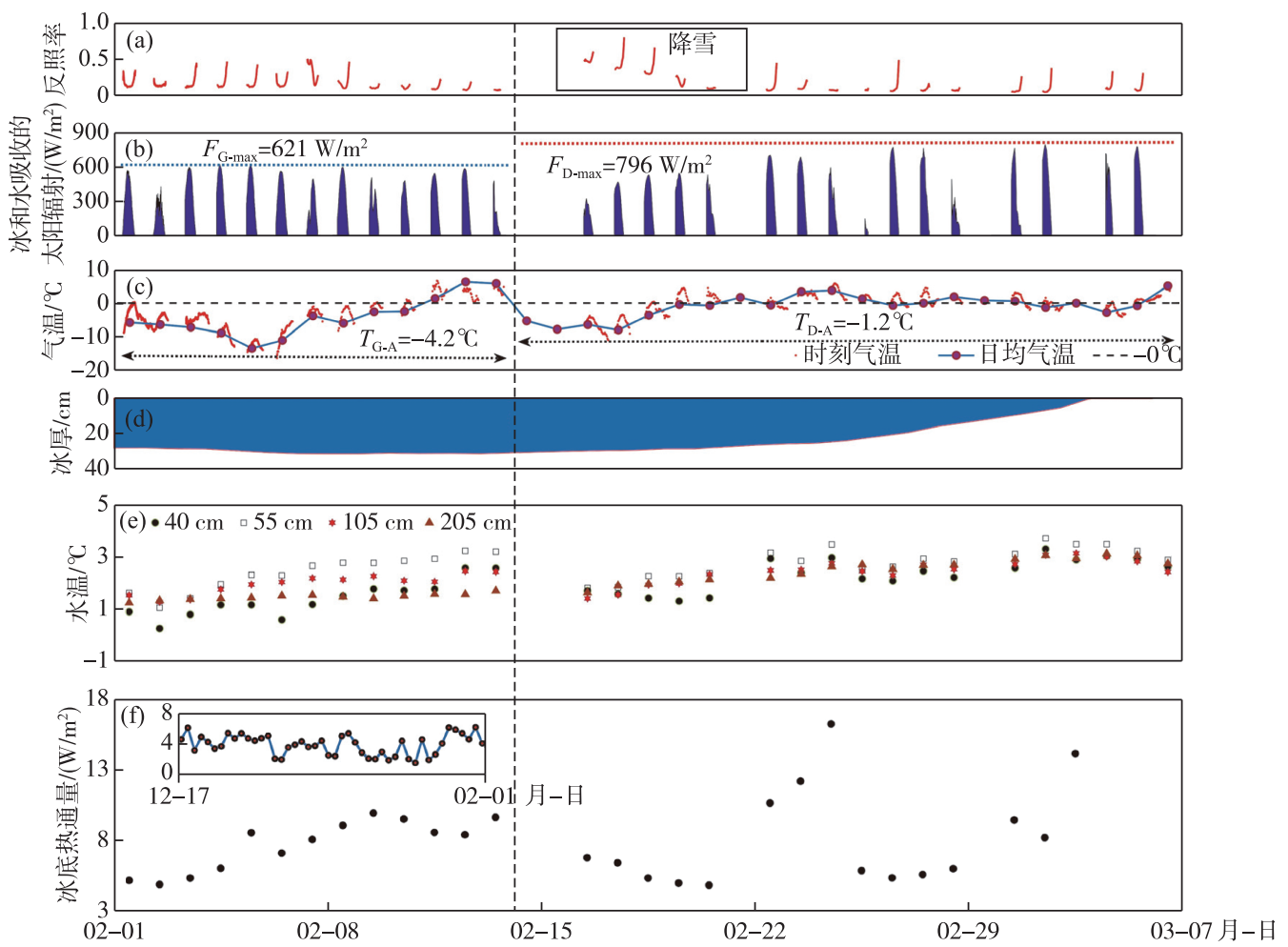

图 7 湖冰消融期气象要素及湖冰特征

( $\mathrm{a}$ : 反照率; $\mathrm{b}$ : 湖冰及冰下水体吸收的太阳辐射 $; \mathrm{c}:$ 气温; $\mathrm{d}$ : 冰厚; $\mathrm{e}$ : 水温; $\mathrm{f}$ : 冰底热通量)

Fig.7 Meteorological elements and evolution of lake ice during the melting period

( a: albedo; b: solar radiation absorbed by lake ice and water; c: air temperature; $\mathrm{d}$ : ice thickness; e: water temperature; $\mathrm{f}$ : water-to-ice heat flux) 
贡献了湖冰消融, 而气温依然在阻碍湖冰消融. 反观冰厚底部发展存在显著的加速衰减过程 (图 7d), 冰下 水温逐渐升高 (图 7e), 且最高水温深度位于 $50 \mathrm{~cm}$ 处, 高于 40 与 $105 \mathrm{~cm}$ 的水温. 基于冰水界面处的温度梯 度利用梯度法 ${ }^{[30]}$ 可以估算冰底热通量 (图 7f), 发现湖冰生长期冰底热通量变化较为平稳, 均值为 $4.8 \mathrm{~W} /$ $\mathrm{m}^{2}$. 而消融期冰底热通量则在 $4.9 \sim 14.1 \mathrm{~W} / \mathrm{m}^{2}$ 较大范围内变化, 均值为 $8.1 \mathrm{~W} / \mathrm{m}^{2}$, 为生长期的 1.7 倍; 且其 峰值均对应于冰下水温的峰值. 同时, 生长期均值与 Jakkila 等 ${ }^{[31]}$ 在芬兰 Pääjärvi 湖以及 Aslamov 等 ${ }^{\left[{ }^{[0]}\right.}$ 在俄 罗斯 Baikal 湖得到的结果一致; 消融期均值与 Leppäranta 等 ${ }^{[32]}$ 在俄罗斯 Vendyurskoe 湖得到的结果吻合.

湖冰消融期的这种快速变化与整个湖面开阔水域的延伸现象密切相关 (图 2). 首先, 湖冰消融期观测 点东侧桥墩处出现的开阔水域造成了整个湖冰侧向边界面的融化行为, 而随着湖冰侧向融化的进行, 开阔 水域的面积也逐渐增大; 其次, 开阔水域的出现导致 $95 \%$ 以上的太阳辐射能量被水体吸收, 高于通常冰下水 体吸收的 30\% 40\% (透射率) 太阳辐射能量; 最后, 开阔水域的水体在温差和风的作用下产生横向热交换, 加之观测点距离开阔水域的距离仅为 $120 \mathrm{~m}$ 左右 (2020 年 2 月 25 日), 因此出现了观测点冰下水温的升高, 间接贡献了冰底的融化. 在整个能量交换中, 冰底融化的能量来源涉及到众多影响因素,例如风速、冰下流 速与水平水温差异的贡献等. 这一正反馈作用解释了图 3e 中冰下水温升高的原因, 也同样是冰厚加速融化 (图 7e) 与冰底热通量升高的根源 (图 7f). 综上, 太阳辐射作为湖冰消融的主要能量来源, 湖冰整体的侧向 融化作为催化剂, 二者共同控制了湖冰的加速融化过程.

\section{5 结论}

1) 2019-2020 年冬季含章湖观测的完整湖冰生消过程共 $99 \mathrm{~d}$, 分为封冻转换期 $3 \mathrm{~d}$ 、稳定生长期 $62 \mathrm{~d}$ 、 冰厚稳定期 $7 \mathrm{~d}$ 、快速消融期 $24 \mathrm{~d}$ 、破碎分解期 $3 \mathrm{~d}$. 湖冰生长过程呈线性趋势, 生长速率为 $0.4 \mathrm{~cm} / \mathrm{d}$, 最大冰 厚为 $30.7 \mathrm{~cm}$; 湖冰消融过程呈抛物型趋势, 消融速率从 $0.3 \mathrm{~cm} / \mathrm{d}$ 增加到 $2.7 \mathrm{~cm} / \mathrm{d}$.

2) 气温是导致冰厚发展的主要因素, 不同深度 $(5 \sim 17 \mathrm{~cm})$ 的冰温响应气温变化存在显著的滞后效应, 滞后时间为 70 158 min, 冰温与气温的最大相关系数为 $0.89 \sim 0.52$; 降雨事件会造成冰上环境湿度显著升高 (趋于 $100 \% \mathrm{RH}$ ) 、冰面反照率从 0.22 降至 0.09 、浅层 $(5 \sim 40 \mathrm{~cm}$ ) 水温升高; 随着降雨停止与气温下降, 湖冰 特征会迅速恢复到降雨前的状态; 降雪事件会造成冰面反照率由 0.25 增至 0.90 , 干扰浅层 $(5 \mathrm{~cm})$ 冰温对气 温的响应效应, 且冰上积雪对气一冰热交换水平的影响具有一定的持续性; 风应力是决定冰上积雪重新分配 的关键因素, 风速大小与持续时间间接影响了冰上积雪对湖冰的阻隔控制.

3) 消融期湖冰的反照率较低, 湖冰与冰下湖水吸收的太阳辐射能量较生长期更大. 湖泊空间差异引发 的开阔水域延伸现象是造成湖冰加速衰减的关键原因, 太阳辐射直接进人开阔水域导致了湖冰侧向界面的 融化, 同时引起了冰下水体与开阔水域之间的横向热交换. 湖冰生长期的冰底热通量均值为 $4.8 \mathrm{~W} / \mathrm{m}^{2}$, 到消 融期增加至 $8.1 \mathrm{~W} / \mathrm{m}^{2}$, 为生长期的 1.7 倍.

4) 相较于以往湖冰观测研究, 利用浮式观测平台获取的湖冰生消过程更加完整, 记录到的气象要素与 水文要素也更加全面, 未来可以依托此手段进一步开展不同区域湖冰生消过程、特别是消融期的监测试验. 湖冰消融期冰底的能量来源较为复杂, 同时包含垂直方向与水平方向的共同热交换作用,有必要对整个湖 冰的侧向融化过程与能量平衡机制进一步开展研究.

致谢: 感谢盘锦市大洼区荣兴街道办事处在观测过程中给予的大力帮助!

\section{6 参考文献}

[ 1 ] Verpoorter C, Kutser T, Seekell DA et al. A global inventory of lakes based on high-resolution satellite imagery. Geophysical Research Letters, 2014, 41(18) : 6396-6402. DOI: 10.1002/2014GL060641.

[ 2 ] Sharma S, Blagrave K, Magnuson JJ et al. Widespread loss of lake ice around the Northern Hemisphere in a warming world. Nature Climate Change, 2019, 9(3) : 227-231. DOI: 10.1038/s41558-018-0393-5.

[ 3 ] Brown LC, Duguay CR. The response and role of ice cover in lake-climate interactions. Progress in Physical Geography: Earth and Environment, 2010, 34(5) : 671-704. DOI: 10.1177/0309133310375653.

[ 4 ] Williams G, Layman KL, Stefan HG. Dependence of lake ice covers on climatic, geographic and bathymetric variables. Cold Regions Science and Technology, 2004, 40(3) : 145-164. DOI: 10.1016/j.coldregions.2004.06.010. 
[ 5 ] Butler TM, Wilhelm AC, Dwyer AC et al. Microbial community dynamics during lake ice freezing. Scientific Reports, 2019, 9(1) : 1-11. DOI : 10.1038/s41598-019-42609-9.

[ 6 ] Hampton SE, Galloway AWE, Powers SM et al. Ecology under lake ice. Ecology Letters, 2017, 20(1) : 98-111. DOI: 10. 1111/ele.12699.

[ 7 ] Liu XZ, Tan YG, Li HS et al. Test and fracture toughness analysis for static ice pressure of reservoir slope. Engineering Mechanics, 2013, 30(5): 112-117, 124. [刘晓洲, 檀永刚, 李洪升等. 水库护坡静冰压力及断裂韧度测试研究. 工 程力学, 2013, 30(5): 112-117, 124.]

[ 8 ] Qiu YB, Wang XX, Ruan YJ et al. Passive microwave remote sensing of lake freeze-thawing over Qinghai-Tibet Plateau. $J$ Lake Sci, 2018, 30(5) : 1438-1449. DOI: 10.18307/2018.0525. [邱玉宝, 王星星, 阮永俭等. 基于星载被动微波遥 感的青藏高原湖冰物候监测方法. 湖泊科学, 2018, 30(5): 1438-1449.]

[ 9 ] Stepanenko VM, Repina IA, Ganbat G et al. Numerical simulation of ice cover of saline lakes. Izvestiya, Atmospheric and Oceanic Physics, 2019, 55(1) : 129-138. DOI: 10.1134/s0001433819010092.

[10] Bai YL, Li HY, Li ZJ. Numerical model for vertical distribution of dissolved oxygen in cold and icy lake ice. Journal of Hydraulic Engineering, 2017, 48(3) : 373-377. [白乙拉, 李慧莹, 李志军. 寒区结冰湖冰盖下溶解氧垂直分布数值 模型. 水利学报, $2017, \mathbf{4 8}(3): 373-377$. ]

[11] Lv HZ, Li CY, Shi XH et al. Pollutant distribution under different conditions in Lake Ulansuhai ice-water system. J Lake Sci, 2015, 27(6) : 1151-1158. DOI: 10.18307/2015.0621. [吕宏洲, 李畅游, 史小红等. 不同条件下乌梁素海污染 物在冰-水体系中分布规律的模拟. 湖泊科学, 2015, 27(6): 1151-1158.]

[12] Reid T, Crout N. A thermodynamic model of freshwater Antarctic lake ice. Ecological Modelling, 2008, 210(3) : 231241. DOI: $10.1016 /$ j.ecolmodel.2007.07.029.

[13] Jeffries MO, Morris K, Duguay CR. Lake ice growth and decay in central Alaska, USA : Observations and computer simulations compared. Annals of Glaciology, 2005, 40: 195-199. DOI: 10.3189/172756405781813807.

[14] Hamilton DP, Magee MR, Wu CH et al. Ice cover and thermal regime in a dimictic seepage lake under climate change. Inland Waters, 2018, 8(3) : 381-398. DOI: 10.1080/20442041.2018.1505372.

[15] Ariano SS, Brown LC. Ice processes on medium-sized north-temperate lakes. Hydrological Processes, 2019, 33 ( 18 ): 2434-2448. DOI: 10.1002/hyp.13481.

[16] Rouse WR, Blanken PD, Bussières N et al. An investigation of the thermal and energy balance regimes of great slave and great bear lakes. Journal of Hydrometeorology, 2008, 9(6) : 1318-1333. DOI: 10.1175/2008jhm977.1.

[17] Cheng B, Vihma T, Rontu L et al. Evolution of snow and ice temperature, thickness and energy balance in Lake Orajärvi, northern Finland. Tellus A: Dynamic Meteorology and Oceanography, 2014, 66 (1): 21564. DOI: 10.3402/tellusa. v66.21564.

[18] Svacina NA, Duguay CR, Brown LC. Modelled and satellite-derived surface albedo of lake ice-Part I: Evaluation of the albedo parameterization scheme of the Canadian Lake Ice Model. Hydrological Processes, 2014, 28 ( 16) : 4550-4561. DOI: 10.1002/hyp. 10253.

[19] Li ZJ, Yang Y, Peng XM et al. The analysis of the field observation data of fresh ice growing process in Hongqipao reservoir of Heilongjiang. Journal of Xi'an University of Technology, 2009, 25(3): 270-274. [李志军, 杨宇, 彭旭明等. 黑 龙江红旗泡水库冰生长过程现场观测数据的剖析. 西安理工大学学报, 2009, 25(3): 270-274. $]$

[20] Tuo YC, Liu ZG, Deng Y et al. Water temperature of the Fengman reservoir with seasonal ice cover. Advances in Water Science, 2014, 25(5): 731-738. [脱友才, 刘志国, 邓云等. 丰满水库水温的原型观测及分析. 水科学进展, 2014, 25 (5) : 731-738.]

[21] Huang WF, Han HW, Niu FJ et al. Field observations on water temperature and stratification in a seasonally ice-covered shallow thermokarst lake. Advances in Water Science, 2016, 27(2) : 280-289. [黄文峰, 韩红卫, 牛富俊等. 季节性冰 封热融浅湖水温原位观测及其分层特征. 水科学进展, 2016, 27(2): 280-289.]

[22] Cao XW, Lu P, Leppäranta M et al. Solar radiation transfer for an ice-covered lake in the central Asian arid climate zone. Inland Waters, 2021, 11(1) : 89-103. DOI: 10.1080/20442041.2020.1790274.

[23] Tang MG, Li ZJ, Lu P et al. Comparison and analysis on the statistical models for the diurnal variations of lake ice albedo under clear skies of Lake Ulansuhai. J Lake Sci, 2020, 32(6) : 1858-1868. DOI: 10.18307/2020.0625. [汤明光, 李志 军, 卢鹏等. 乌梁素海湖冰晴天反照率日变化特征的统计模型比较和分析. 湖泊科学, $2020,32(6): 1858-1868$. ] 
[24] Kirillin G, Leppäranta M, Terzhevik A et al. Physics of seasonally ice-covered lakes: A review. Aquatic Sciences, 2012, 74(4) : 659-682. DOI: 10.1007/s00027-012-0279-y.

[25] Liu TY, Liu ZB, Zhu H et al. Analysis on the Change Trend of Winter Temperature in Yingkou City from 1951 to 2013. Modren Agricultural Science and Technology 2015, 4(17): 286-291. [刘桐义, 刘志邦, 朱红等. 1951-2013 年营口市 冬季气温变化趋势分析. 现代农业科技, 2015, 4(17): 286-291.]

[26] Stefan J. Ueber Die Theorie der Eisbildung, insbesondere über Die Eisbildung im Polarmeere. Annalen Der Physik, 1891, 278(2) : 269-286. DOI: $10.1002 /$ andp.18912780206.

[27] Lei RB, Li ZJ, Zhang ZH et al. Comparisons of thermodynamic processes between lake ice and landfast sea ice around Zhongshan station, east Antarctica. Chinese Journal of Polar Research, 2011, 23 (4) : 289-298. DOI: 10.3724/SP. J. 1084.2011.00289. [雷瑞波, 李志军, 张占海等. 东南极中山站附近湖冰与固定冰热力学过程比较. 极地研究, $2011,23(4)$ : 289-298.]

[28 ] Lu P, Leppäranta M, Cheng B et al. Influence of melt-pond depth and ice thickness on Arctic sea-ice albedo and light transmittance. Cold Regions Science and Technology, 2016, 124: 1-10. DOI: 10.1016/j.coldregions.2015.12.010.

[29] Huang WF, Zhang JR, Leppäranta M et al. Thermal structure and water-ice heat transfer in a shallow ice-covered thermokarst lake in central Qinghai-Tibet Plateau. Journal of Hydrology, 2019, 578: 124122. DOI: 10. 1016/j. jhydrol. 2019.124122.

[30] Aslamov IA, Kozlov VV, Kirillin GB et al. Ice-water heat exchange during ice growth in Lake Baikal. Journal of Great Lakes Research, 2014, 40(3) : 599-607. DOI: 10.1016/j.jglr.2014.06.004.

[31] Jakkila J, Leppäranta M, Kawamura T et al. Radiation transfer and heat budget during the ice season in Lake Päajjärvi, Finland. Aquatic Ecology, 2009, 43(3) : 681-692. DOI: 10.1007/s10452-009-9275-2.

[32] Leppäranta M, Terzhevik A, Shirasawa K. Solar radiation and ice melting in Lake Vendyurskoe, Russian Karelia. Hydrology Research, 2010, 41(1) : 50-62. DOI: 10.2166/nh.2010.122. 